\title{
Patterns of Plasma Fatty Acids in Rat Models with Adenovirus Infection
}

\author{
Man Jeong Paik ${ }^{1, \#, ~ K i ~ H o ~ P a r k ~}{ }^{2, \#}$, Joong Jean Park³, Kyoung Rae Kim, \\ Young Hwan Ahn ${ }^{4}$, Gyu Tae Shin ${ }^{5}$ and Gwang Lee ${ }^{6,7,8, *}$ \\ ${ }^{1}$ Biometabolite Analysis Laboratory, College of Pharmacy, \\ ${ }^{2}$ Department of Molecular Cell Biology, Sungkyunkwan University, Suwon 440-746, Korea \\ ${ }^{3}$ Department of Physiology, College of Medicine, Korea University, Seoul 136-705, Korea \\ ${ }^{4}$ Department of Neurosurgery and ${ }^{5}$ Department of Nephrology and ${ }^{6}$ Brain Disease Research Center and \\ ${ }^{7}$ Department of Molecular Science and Technology and ${ }^{8}$ Institute for Neuroregeneration and Stem Cell Research, \\ Ajou University School of Medicine, Suwon 442-721, Korea
}

Received 21 July 2006, Accepted 6 September 2006

\begin{abstract}
Adenoviral vectors are among the most promising vectors available for human gene therapy. However, the use of recombinant adenoviral vectors, including replicationcompetent adenovirus (RCA), raises a variety of safety concerns in relation to the development of new therapies based on gene therapy. To examine how organic compounds change in rat plasma following the injection of adenovirus, $\beta$-galactosidase expressing recombinant adenovirus (designated rAdLacZ) or RCA, we investigated the content of fatty acids (FAs), which are important biochemical indicators in pathological conditions. Pattern recognition analysis on the level of FAs in rat plasma is described for the visual discrimination of adenovirus infection groups from normal controls. Plasma FAs from four control rats (normal group), and from four rats with rAdLacZ infection and six rats with RCA infection (the two abnormal groups), were examined by gas chromatography-mass spectrometry in selected ion monitoring modes as their tert-butyldimethylsilyl derivatives. In total, 20 FAs were positively detected and quantified. The results of the Student's $t$-test on the normal mean of two abnormal groups, the levels of three FAs $(p<0.05)$ from rAdLacZ group and eleven FAs $(p<0.05)$ from RCA group were significantly different. When star symbol plotting was applied to the group mean values of 20 FAs after normalization to the corresponding normal mean values, the resulting eicosagonal star patterns of the two infected groups were distorted into similar shapes, but were distinguishable from each other. Thus, these approaches
\end{abstract}

${ }^{\#}$ These authors contributed equally to this work.

*To whom correspondence should be addressed.

Tel: 82-31-219-4554; Fax: 82-31-216-6381

E-mail: glee@ajou.ac.kr will be useful for screening and monitoring of diagnostic markers for the effects of infection following the use of adenoviral vectors in gene therapy.

Keywords: Adenovirus, Fatty acids, Gas chromatographymass spectrometry, Infection, Star symbol plot

\section{Introduction}

Recombinant adenoviral vectors have been used to deliver foreign genes into diverse cell types and a wide variety of tissues (Wilson, 1996; Baum et al., 2003; Kesisoglou et al., 2005) and have been studied for use in live viral vaccines and for human gene therapy (Ye et al., 1991; Ferreira et al., 2005; Campochiaro et al., 2006; Spearman, 2006). Although adenoviral vectors have been proven to be useful for gene transfer in vitro and in vivo, several limitations remained to understand, including the hazards of viral recombination and the interactions of adenoviral vectors with immune systems. Adenoviral vectors from which the E1 region has been deleted are defective in replication. In some cases, E1-deleted viruses can replicate in infected cells, which is believed to trigger a deleterious cellular immune response (Liu and Muruve, 2003). However, a few studies have investigated the effects of infection by these vectors.

To determine how organic compounds change following the injection of adenoviruses into rat models, fatty acids (FAs) were investigated in plasma infected by adenoviruses, because FAs occurring in metabolic pathways are important biochemical indicators for pathological conditions (Okun et al., 2002; Shigematsu et al., 2003; Olpin, 2004). Moriya et al. reported the association of FAs with viral infection with hepatitis $\mathrm{C}$ (Moriya et al., 2001). The authors found that the proportion of 
FAs such as oleic and vaccenic acids significantly increased in hepatitis $\mathrm{C}$ infected livers and suggested that viral infection may affect a specific pathway in lipid metabolism. In the case of adenoviral vectors, lipid metabolism following infection by these viruses have not been studied.

Therefore, studying these compounds is important for biochemical diagnoses in the clinical field. The simultaneous analysis of multiple FAs in unknown complex biosamples requires the use of high-resolution gas chromatography (GC) combined with mass spectrometry (MS). Our previous GC profiling analysis of tert-butyldimethylsilyl (TBDMS) derivatives provided rapid screening for organic acids including FAs in aqueous samples (Paik and Kim, 2004; Paik et al., 2005).

The accurate discrimination of normal states from abnormal states requires the application of a proper pattern recognition method for the complex metabolic profiles. In our previous reports, star symbol plotting as the visual pattern recognition method was found to be useful in comparative analyses (Kim et al., 1997). When it was applied to very long chain FAs in plasma, an X-linked adrenoleukodystrophy patient group was readily discriminated from the normal control group (Paik et al., 2001). FA compositions of plasma are well documented in the literature (Costa et al., 1998; Yoon et al., 2000; Paik et al., 2001), but attempts have seldom been made to correlate the FA profiles to adenoviral-induced physiological changes.

In the experiments described in this report, GC-MS analysis of plasma FAs as TBDMS derivatives in selected ion monitoring (SIM) mode was combined with star symbol plotting for a visual comparison between a normal control group and two viral infection groups treated with $\beta$-galactosidase expressing recombinant adenovirus (rAdLacZ) or replication-competent adenovirus (RCA). The two infection groups showed changes in FA levels compared with normal group mean values that were subjected to star symbol plotting after normalization to their respective normal mean values. Thus, these results revealed that the monitoring of FAs in adenovirus infections will be useful as a biochemical diagnostic marker.

\section{Materials and Methods}

Cell cultures. Human embryonic kidney cells (HEK 293 cells) and human alveolar basal epithelial cells (A549 cells) were obtained from the Korean Cell Line Bank and grown in Dulbecco's modified Eagle's medium supplemented with $10 \%$ (vol/vol) fetal bovine serum (FBS).

Adenoviruses and viral assay methods. For this study, two types of adenovirus were used. rAdLacZ (Shin et al., 2005) expresses the Escherichia coli lacZ gene coding $\beta$-galactosidase, and RCA (adenovirus type 5) was obtained from the American Type Culture Collection (ATCC VR-1516). The infectious titer of RCA on HEK 293 cells was $7 \times 10^{10}$ NAS Infectious Units (NIU)/ml as a reference. rAdLacZ was propagated in HEK 293 cells and purified using an Adeno-XIM Virus Purification Kit (BD Biosciences), and then concentrated using Centricon TM-50 centrifugal filter units
(Millipore) and titrated in HEK 293 cells using an Adeno-XTM Rapid Titer Kit (BD Biosciences). Adenoviral genomic DNA was extracted from the purified rAdLacZ or RCA following the addition of Proteinase K (Sigma), phenol-chloroform extraction, and ethanol precipitation. To check the contamination of RCA in rAdLacZ, the following primers were used: 5'-TATGGGCAGTGGGTGATA-3', 5'-GCGTTTACAGCTCAAGTC-3'. Samples were amplified with initial denaturation at $94^{\circ} \mathrm{C}$ for $5 \mathrm{~min}$, followed by 30 cycles of denaturation at $94^{\circ} \mathrm{C}$ for $1 \mathrm{~min}$, annealing at $55^{\circ} \mathrm{C}$ for $1 \mathrm{~min}$, extension at $72^{\circ} \mathrm{C}$ for $1 \mathrm{~min}$, and a final extension at $72^{\circ} \mathrm{C}$ for $5 \mathrm{~min}$. Amplified products were separated by agarose gel (1\%) electrophoresis in Tris-acetate-ethylenediaminetetra-acetate (TAE) buffer, and DNA fragments were visualized by ethidium bromide staining.

Animals and plasma specimens. Sprague-Dawley rats (250-300 g) were used and infected with rAdLacZ or RCA. Approximately $1 \times 10^{10}$ plaque-forming units $(\mathrm{pfu})$ in $0.1 \mathrm{ml}$ total volume of phosphate-buffered saline (PBS) were injected into a tail vein. Animals were briefly anesthetized prior to injection with Chloral Hydrate Solution (Fluka). Plasma samples were collected from four normal control rats, four rats infected with rAdLacZ virus, and six rats infected with RCA virus at $72 \mathrm{~h}$ after exposure to the virus. All samples were immediately stored at $-70^{\circ} \mathrm{C}$ until analyzed.

Chemicals and reagents. The 20 FA standards, pentadecanoic acid as internal standard and triethylamine were purchased from Sigma. $\mathrm{N}$-methyl- $\mathrm{N}$-(tert-butyldimethylsilyl) trifluoroacetamide (MTBSTFA) was obtained from Pierce. Acetonitrile, toluene, diethyl ether, and dichloromethane of pesticide grade were obtained from Kanto Chemical. Sodium chloride was purchased from Junsei and washed successively with methanol, acetone, dichloromethane, and diethyl ether, followed by drying under a vacuum $\left(100^{\circ} \mathrm{C}, 1 \mathrm{~h}\right)$. Sulfuric acid and sodium hydroxide were obtained from Duksan.

Gas chromatography-mass spectrometry. For the quantitative analysis of FAs in rat plasma, GC-MS analysis in selected ion monitoring (SIM) mode was conducted with an Agilent 6890 gas chromatograph, interfaced to an Agilent 5973 mass-selective detector (70 eV, electron impact mode) and installed with an Ultra-2 (SE-54 bonded phase; $25 \mathrm{~m} \times 0.20 \mathrm{~mm}$ I.D., $0.11 \mu \mathrm{m}$ film thickness) crosslinked capillary column (Agilent Technologies). Helium was used as carrier gas at a flow rate of $0.5 \mathrm{ml} / \mathrm{min}$ in constant flow mode. The injector, interface, and ion source were maintained at 260, 300, and $230^{\circ} \mathrm{C}$, respectively. Samples were introduced in the splitinjection mode $(10: 1)$. The oven temperature was set initially at $100^{\circ} \mathrm{C}(2 \mathrm{~min})$ and then programmed to increase at a rate of $3^{\circ} \mathrm{C} /$ min to $260^{\circ} \mathrm{C}$, and finally at $20^{\circ} \mathrm{C} / \mathrm{min}$ to $300^{\circ} \mathrm{C}(10 \mathrm{~min})$.

Sample preparation. FAs were converted into their TBDMS derivatives according to our previous method (Paik and Kim, 2004; Paik et al., 2005). Briefly, $1 \mathrm{ml}$ of acetonitrile was added to $0.2 \mathrm{ml}$ of plasma to remove protein. After centrifugation, pentadecanoic acid $(5.0 \mu \mathrm{g})$ as the internal standard and $1 \mathrm{ml}$ of distilled water were added to the supernatant, and the $\mathrm{pH}$ was adjusted to 12 using 5.0 M sodium hydroxide, with subsequent washing with diethyl ether $(3 \mathrm{ml} \times 2)$. The aqueous phase was then acidified $(\mathrm{pH} \leq 2.0$ with concentrated sulfuric acid) and saturated with sodium chloride, followed by extraction with diethyl ether $(3 \mathrm{ml} \times 2)$. The extracts 
were evaporated to dryness under a gentle nitrogen stream. The residues containing FAs were reacted $\left(60^{\circ} \mathrm{C}\right.$ for $\left.30 \mathrm{~min}\right)$ with MTBSTFA $(20 \mu \mathrm{l})$ in toluene $(30 \mu \mathrm{l})$ containing triethylamine $(5 \mu \mathrm{l})$ to form TBDMS derivatives for analysis by GC-MS in SIM mode. All samples were individually prepared in triplicate.

Pattern recognition analysis. The levels of FAs in each sample were measured using calibration curves. The FA levels of each infected sample were normalized to the corresponding normal median values. The levels of 20 FAs after normalization to the corresponding normal mean values were used as the variables for star symbol plotting. Each value was plotted as a line radiating from a common central point, and the far ends of the lines were joined together to produce group star patterns using MS Excel, as described elsewhere (Kim et al., 1997; Paik et al., 2001).

\section{Results and Discussion}

Detection of RCA in purified rAdLacZ. To exclude the possibility of RCA contamination in purified rAdLacZ, we used PCR methods and a cytopathic effect (CPE) assay. To detect contamination of RCA in purified rAdLacZ, we amplified a 410-bp fragment spanning portions of the E1 region (Fig. 1A). The E1 fragment was only detected in the RCA lane (Fig. 1A, lane 2). Since A549 cells are highly sensitive to RCA, we used these cells for the CPE assay with $100 \mathrm{pfu} /$ dish. A CPE was observed only in RCA, but not in rAdLacZ (Fig. 1B). Even when we increased the concentration to 1,000 $\mathrm{pfu} /$ dish in rAdLacZ, a CPE was not detected (data not shown). The observation that RCA in rAdLacZ was not detected by PCR or the CPE assay indicates purified rAdLacZ that does not contain RCA. Therefore, we used rAdLacZ and RCA for further study.

Plasma fatty acid levels in viral infection gropus and star pattern recognition. To analysis how organic compounds change following the infection of adenoviruses, rAdLacZ or $\mathrm{RCA}$, we injected about $1 \times 10^{10}$ pfu of adenovirus into a tail vein of rat. Animals were sacrificed 3 days after the injection and the quantity of FAs was determined in the individual plasma. In total, 20 FAs were positively detected from 14 plasma samples. Large variations in their levels were observed within each group. According to the Student's $t$-test on the mean values of normal and two viral infection groups, the levels of palmitic acid $(p<0.05)$, oleic acid $(p<0.04)$ and stearic acid $(p<0.03)$ were significantly elevated in rAdLacZ group while the levels of myristic acid $(p<0.04)$, palmitoleic acid $(p<0.03)$, palmitic acid $(p<0.03)$, linoleic acid $(p<$ $0.04)$, oleic acid $(p<0.004)$, linolenic acid $(p<0.03)$, stearic acid $(p<0.05)$, eicosanoic acid $(p<0.01)$, behenic acid $(p<0.03)$, nervonic acid $(p<0.03)$ and hexacosanoic acid $(p<0.0005)$ were elevated in RCA group as compared to normal subjects (Table 1).

When the FA levels in each sample of rAdLacZ and RCA groups were normalized to the corresponding normal mean
(A)

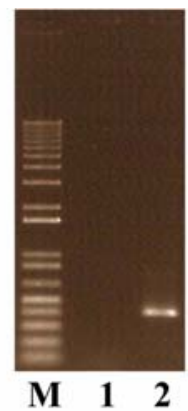

(B)

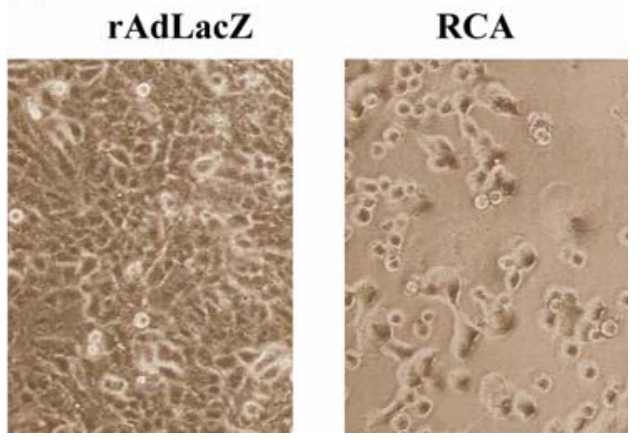

Fig. 1. Detection of RCA in rAdLacZ by PCR and a CPE assay. A panel; E1 fragment DNA derived from RCA was detected by PCR. M, 1-kb ladder marker (Gibco BRL); lane 1, rAdLacZ; lane 2, RCA. B panel; RCA was detected by a CPE assay. A549 cells were infected with RCA or rAdLacZ with 100 pfu/dish. A CPE was observed on day 6.

values they proved very informative by expressing the elevation of the FA levels in multiples (ranging from 0.751 to 2.372) of the normal mean values (Table 1). In the rAdLacZ group, oleic acid (No. 10) showed the highest value, the second prominent FA was linolenic acid (No. 11), followed by palmitoleic acid (No. 7). While in the RCA group, palmitoleic acid (No. 7) showed the highest value, the second prominent FA was oleic acid (No. 10), followed by linolenic acid (No. 11). The eicosanoic acid (No. 15) in the RCA infection group were much larger, as compared to the rAdLacZ group. No notable differences between the two infection groups in the levels of other FAs except for lauric acid (No. 5), myristic acid (No. 6) and palmitoleic acid (No. 7) were observed. Seven FAs (Nos. 7 through 12 and No. 15) showed changes in their levels by more than $40 \%$ from the normal mean values. The star symbol plots drawn based on these values displayed distorted eicosagonal shapes for the rAdLacZ group (Fig. 2A) and the RCA group (Fig. 2B), as compared to the pattern of the normal group.

It is noteworthy that, although the pattern of the RCA group (Fig. 2B) was similar to that of the rAdLacZ group (Fig 2A) in the star symbol plots except for the level of eicosanoic acid (No. 15), the size of the RCA group was larger than that of the rAdLacZ group. This may explain why RCA displayed more distorted eicosagonal shapes for infected groups, as compared 
Table 1. Normalized values of fatty acids in plasma samples from four rats infected with rAdLacZ and six rats infected with RCA

\begin{tabular}{|c|c|c|c|c|}
\hline \multirow{3}{*}{ Fatty acids } & \multicolumn{4}{|c|}{ Fatty acid value ${ }^{a}$} \\
\hline & \multicolumn{2}{|c|}{ A group ${ }^{b}$} & \multicolumn{2}{|c|}{$\mathrm{R}$ group $^{\mathrm{c}}$} \\
\hline & mean $\pm \mathrm{SD}$ & $p$-value ${ }^{\mathrm{d}}$ & mean $\pm \mathrm{SD}$ & $p$-value ${ }^{\mathrm{d}}$ \\
\hline 1. Caproic acid & $0.824 \pm 0.193$ & NS & $0.791 \pm 0.182$ & NS \\
\hline 2. Caprylic acid & $0.835 \pm 0.262$ & NS & $0.751 \pm 0.201$ & NS \\
\hline 3. Decenoic acid & $0.994 \pm 0.039$ & NS & $0.978 \pm 0.023$ & NS \\
\hline 4. Capric acid & $0.874 \pm 0.204$ & NS & $0.855 \pm 0.151$ & NS \\
\hline 5. Lauric acid & $0.985 \pm 0.141$ & NS & $1.217 \pm 0.363$ & NS \\
\hline 6. Myrisic acid & $1.071 \pm 0.103$ & NS & $1.289 \pm 0.260$ & 0.04 \\
\hline 7. Palmitoleic acid & $1.855 \pm 1.130$ & NS & $2.372 \pm 1.188$ & 0.03 \\
\hline 8. Palmitic acid & $1.528 \pm 0.395$ & 0.05 & $1.524 \pm 0.374$ & 0.03 \\
\hline 9. Linoleic acid & $1.551 \pm 0.543$ & NS & $1.569 \pm 0.525$ & 0.04 \\
\hline 10. Oleic acid & $2.013 \pm 0.957$ & 0.04 & $2.200 \pm 0.630$ & 0.004 \\
\hline 11. Linolenic acid & $1.997 \pm 1.016$ & NS & $2.193 \pm 0.983$ & 0.03 \\
\hline 12. Stearic acid & $1.323 \pm 0.145$ & 0.03 & $1.413 \pm 0.383$ & 0.05 \\
\hline 13. Eicosatetraenoic acid & $1.078 \pm 0.178$ & NS & $1.163 \pm 0.441$ & NS \\
\hline 14. Eicosapentaenoic acid & $1.179 \pm 0.296$ & NS & $1.222 \pm 0.244$ & NS \\
\hline 15. Eicosanoic acid & $1.026 \pm 0.099$ & NS & $1.522 \pm 0.342$ & 0.01 \\
\hline 16. Docsapentaenoic acid & $1.101 \pm 0.360$ & NS & $1.134 \pm 0.298$ & NS \\
\hline 17. Behenic acid & $0.822 \pm 0.003$ & NS & $0.827 \pm 0.005$ & 0.03 \\
\hline 18. Nervonic acid & $1.002 \pm 0.004$ & NS & $1.003 \pm 0.002$ & 0.03 \\
\hline 19. Lignoceric acid & $1.000 \pm 0.001$ & NS & $1.002 \pm 0.003$ & NS \\
\hline 20. Hexacosanoic acid & $0.994 \pm 0.018$ & NS & $0.971 \pm 0.002$ & 0.0005 \\
\hline
\end{tabular}

All samples were individually prepared in triplicate.

${ }^{a}$ Values normalized to corresponding normal mean values

${ }^{\mathrm{b}}$ Group infected with rAdLacZ

${ }^{\circ}$ Group infected with RCA

${ }^{\mathrm{d}}$ Student's $t$-test at $95 \%$ confidence level

NS, not significantly different

to the pattern of the rAdLacZ group, since RCA has direct toxicity and can cause inflammation in the host by activating inflammatory gene expression (Smith et al., 1993; Liu and Muruve, 2003).

Adenoviral vector administration led to induce the elaboration of interleukin (IL)-6, 12, macrophage inflammatory protein (MIP)-2 and tumor necrosis factor (TNF)-a in serum, play an important role in the pathological conditions (Varnavski et al., 2005). The relevance of the change of FAs and immune responses associated with injection of adenovirus has not been studied. However, it is noteworthy that free FA, palmitate is responsible for the activation of IL-6 gene expression in human myotubules (Weigert et al., 2004). Therefore, the change of FAs might be related to the immune responses. The association between the change of FAs and immune responses is not thoroughly understood yet, but the approaches of these two studies could be useful for screening and monitoring of the adverse effects associated with infection of adenoviral vectors for gene therapy.

The relevance of the change of FAs and the pathological response after infection with adenovirus has not been studies.
However, the level of FAs, such as oleic and vaccenic acids, which contributes to membrane fluidity, leading to increased cell metabolism, significantly increased in the hepatitis $\mathrm{C}$ infected livers (Moriya et al., 2001). As the hepatitis C infection, adenoviral infection may increase FAs in plasma by a specific lipid pathway. The discovery that adenovirus infection can affect a pathway in lipid metabolism, and thus change FAs state, is essential to our understanding of responses to adenoviral infection. Elucidation of the lipid metabolism by adenovirus infection is needed further investigation.

The star symbol plots, based on the plasma levels of 20 FAs after normalization to the corresponding normal mean values, were found to be effective for the visual recognition of rAdLacZ and RCA groups in distorted eicosagonal patterns. Even if the relevance of the contents of FAs and adenovirus infection was well recognized in this study, other factors, such as diet, are thought to be significant factors because dietary FAs modulate plasma and intracellular cholesterol concentrations (Dorfman and Lichtenstein, 2006). To exclude the variation of diet and other factors in this study, we used four control rats which were fed the same diet. Although the normal group 
(A)

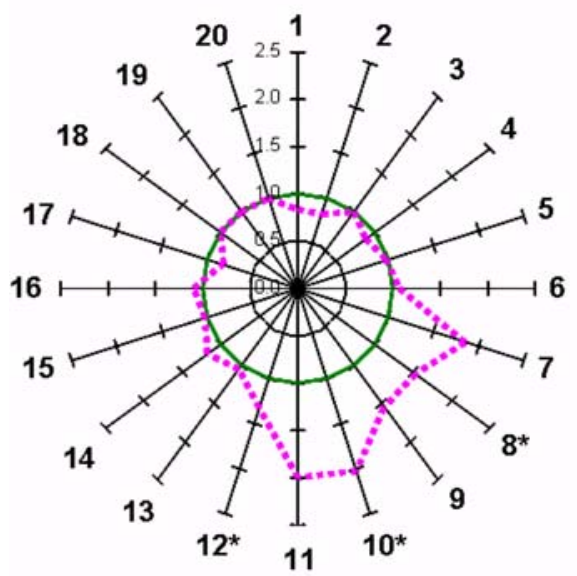

(B)

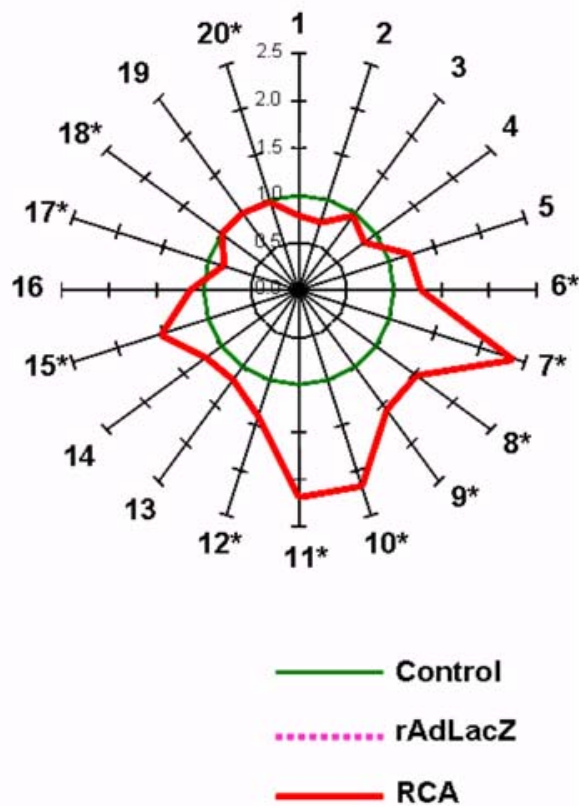

Fig. 2. Star symbol plots of rAdLacZ (A panel), RCA (B panel), and control groups based on the mean levels of the 20 fatty acid variables after normalization to the corresponding normal mean values.

*The results for Student's $t$-test at $95 \%$ confidence level were significantly different.

Rays: the numbers correspond to those in Table 1.

mean served well as the control pattern for the rAdLacZ and RCA groups, there is a pressing need for large-scale studies of fatty acids in the context of adenoviruses for further attempts to clarify the significance of the FAs change in infection with adenovirus. This star symbol plotting also could be useful for visual monitoring and screening for other viral infections.

Acknowledgments This work was supported by the Korea Health 21 R\&D project, Ministry of Health and Welfare, Republic of Korea (0412-DB00-0101-0007) and Korea Food and Drug Administration (04092-005).

\section{References}

Baum, B. J., Goldsmith, C. M., Kok, M. R., Lodde, B. M., van Mello, N. M., Voutetakis, A., Wang, J., Yamano, S. and Zheng, C. (2003) Advances in vector-mediated gene transfer. Immunol. Lett. 90, 145-149.

Campochiaro, P. A., Dong, N. Q., Mahmood, S. S., Klein, M. L., Holz, E., Frank, R. N., Saperstein, D. A., Gupta, A., Stout, J. T., Macko, J. and Dibartolomeo, R. (2006) Adenoviral vectordelivered pigment epithelium-derived factor for neovascular age-related macular degeneration: results of a phase I clinical trial. Hum. Gene Ther. 2, 167-176.

Costa, C. G., Dorland, L., Holwerda, U., de Almeida, I. T., PollThe, B. T., Jakobs, C. and Duran, M. (1998) Simultaneous analysis of plasma free fatty acids and their 3-hydroxy analogs in fatty acid beta-oxidation disorders. Clin. Chem. 44, 463-471.
Dorfman, S. E. and Lichtenstein, A. H. (2006 ) Dietary fatty acids differentially modulate messenger RNA abundance oflowdensity lipoprotein receptor, 3-hydroxy-3-methylglutaryl coenzyme A reductase, and microsomal triglyceride transfer protein in Golden-Syrian hamsters. Metabolism. 55, 635-641.

Ferreira, T. B., Alves, P. M., Aunins, J. G. and Carrondo, M. J. (2005) Use of adenoviral vectors as veterinary vaccines. Gene. Ther. 12, 73-83.

Kesisoglou, F., Chamberlain, J. R., Schmiedlin-Ren, P., Kaz, A., Fleisher, D., Roessler, B. and Zimmermann, E. M. (2005) Chimeric Ad5 vectors expressing the short fiber of Ad41 show reduced affinity for human intestinal epithelium. Mol. Pharm. 2, 500-508.

Kim, K. R., Kim, J. H., Jeong, D. H., Paek, D. J. and Liebich, H. M. (1997) Gas chromatographic profiling analysis of urinary organic acids from nonsmokers and smokers. J. Chromatogr. B. 701, 1-8.

Kim, K. R., Paik, M. J., Kim, J. H., Dong, S. W. and Jeong, D. H. (1997) Rapid gas chromatographic profiling and screening of biologically active amines. J. Pharm. Biomed. Anal. 15, 1309-1318.

Liu, Q. and Muruve, D. A. (2003) Molecular basis of the inflammatory response to adenovirus vectors. Gene. Ther. 10, 935-940.

Moriya, K., Todoroki, T., Tsutsumi, T., Fujie, H., Shintani, Y., Miyoshi, H., Ishibashi, K., Takayama, T., Makuuchi, M., Watanabe, K., Miyamura, T., Kimura, S. and Koike, K. (2001) Increase in the concentration of carbon 18 monounsaturated fatty acids in the liver with hepatitis C: analysis in transgenic mice and humans. Biochem. Biophys. Res. Commun. 281, 1207-1212. 
Okun, J. G., Kolker, S., Schulze, A., Kohlmuller, D., Olgemoller, K., Lindner, M., Hoffmann, G F., Wanders, R. J. and Mayatepek, E. (2002) A method for quantitative acylcarnitine profiling in human skin fibroblasts using unlabelled palmitic acid: diagnosis of fatty acid oxidation disorders and differentiation between biochemical phenotypes of MCAD deficiency. Biochim. Biophys. Acta 1584, 91-98.

Olpin, S. E. (2004) Implications of impaired ketogenesis in fatty acid oxidation disorders. Prostaglandins Leukot. Essent. Fatty Acids 70, 293-308.

Paik, M. J., Kim, K. R., Yoon, H. R. and Kim, H. J. (2001) Diagnostic patterns of very-long-chain fatty acids in plasma of patients with X-linked adrenoleukodystrophy. J. Chromatogr. B 760, 149-157.

Paik, M. J. and Kim, K. R. (2004) Sequential ethoxycarbonylation, methoximation and tert-butyldimethylsilylation for simultaneous determination of amino acids and carboxylic acids by dualcolumn gas chromatography. J. Chromatogr. A 1034, 13-23.

Paik, M. J., Lee, H. J. and Kim, K. R. (2005) Simultaneous retention index analysis of urinary amino acids and carboxylic acids for graphic recognition of abnormal state. J. Chromatogr. B 821, 94-104.

Shigematsu, Y., Hirano, S., Hata, I., Tanaka, Y., Sudo, M., Tajima, T., Sakura, N., Yamaguchi, S., and Takayanagi, M. (2003) Selective screening for fatty acid oxidation disorders by tandem mass spectrometry: difficulties in practical discrimination. $J$. Chromatogr. B 792, 63-72.

Shin, G. T., Kim, W. H., Yim, H., Kim, M. S. and Kim, H. (2005) Effects of suppressing intrarenal angiotensinogen on renal transforming growth factor-beta1 expression in acute ureteral obstruction. Kidney Int. 67, 897-908.

Smith, T. A., Mehaffey, M. G., Kayda, D. B., Saunders, J. M., Yei, S., Trapnell, B. C., McClelland, A. and Kaleko, M. (1993) Adenovirus mediated expression of therapeutic plasma levels of human factor IX in mice. Nat. Genet. 5, 397-402.

Spearman, P. (2006) Current progress in the development of HIV vaccines. Curr. Pharm. Des. 12, 1147-1167.

Varnavski, A. N., Calcedo, R., Bove, M., Gao, G. and Wilson, J. M. (2005) Evaluation of toxicity from high-dose systemic administration of recombinant adenovirus vector in vector-naive and pre-immunized mice. Gene Ther. 12, 427-436.

Weigert, C., Brodbeck, K., Staiger, H., Kausch, C., Machicao, F., Haring, H. U. and Schleicher, E. D. (2004) Palmitate, but not unsaturated fatty acids, induces the expression of interleukin-6 in human myotubes through proteasome-dependent activation of nuclear factor-kappaB. J. Biol. Chem. 279, 23942-23952.

Wilson, J. M. (1996) Adenoviruses as gene-delivery vehicles. $N$. Engl. J. Med. 334, 1185-1187.

Ye, W. W., Mason, B. B., Chengalvala, M., Cheng, S. M., Zandle, G., Lubeck, M. D., Lee, S. G., Mizutani, S., Davis, A. R. and Hung, P. P. (1991) Co-expression of hepatitis B virus antigens by a non-defective adenovirus vaccine vector. Arch. Virol. 118, 11-27.

Yoon, H. G., Yang, S. W., Kim, H. Y., Kim, H. K., Shin, D. H., Hong, B. S. and Cho, H. Y. (2000) Analysis of essential leucine residue for catalytic activity of novel thermostable chitosanase by site-directed mutagenesis. J. Protein Chem. 19, 621-630. 